\title{
Intraoperative monitoring with visual evoked potentials for brain surgeries
}

\author{
Eveline M. Gutzwiller, MD, ${ }^{1}$ Ivan Cabrilo, MD, ${ }^{1}$ Ivan Radovanovic, MD, ${ }^{2}$ Karl Schaller, MD, ${ }^{1}$ and \\ Colette Boëx, $\mathrm{PhD}^{3}$
}

\begin{abstract}
Departments of ${ }^{1}$ Neurosurgery and ${ }^{3}$ Neurology, Geneva University Hospitals and Faculty of Medicine, Geneva, Switzerland; and 2Division of Neurosurgery, Toronto Western Hospitals, Krembil Neuroscience Center, University Health Network and University of Toronto, Ontario, Canada
\end{abstract}

OBJECTIVE The goal of this study was to determine the performance of intraoperative visual evoked potentials (VEPS) in detecting visual field changes.

METHODS Assessments of VEPs were performed with simultaneous retinal responses by using white light-emitting diodes protected from scialytic microscope lights. The alarm criterion was a reproducible decrease in amplitude of the VEP P100 wave of $20 \%$ or more. Visual fields were assessed preoperatively and 1 month postsurgery (Goldmann perimetry).

RESULTS The VEPs were analyzed for 29 patients undergoing resection of a brain lesion. In $89.7 \%$ of patients, steady VEP and retinal responses were obtained for monitoring. The absence of alarm was associated in $94.4 \%$ of cases with the absence of postoperative visual changes (specificity). The alarms correctly identified $66.7 \%$ of cases with any postoperative changes and $100 \%$ of cases with changes more severe than just a discrete quadrantanopia or deterioration of an existing quadrantanopia (sensitivity, new diffuse deterioration $<2 \mathrm{~dB}$ ). In $11.5 \%$ of patients, a transitory VEP decrease with subsequent recovery was observed without postoperative defects.

CONCLUSIONS Intraoperative VEPs were performed with simultaneous recording of electroretinograms, with protection from lights of the operating room and with white light-emitting diodes. Intraoperative VEPs were shown to be reliable in predicting postoperative visual field changes. In this series of intraaxial brain procedures, reliable intraoperative VEP monitoring was achieved, allowing at minimum the detection of new quadrantanopia. The standardization of this technique appears to be a valuable effort in regard to the functional risks of homonymous hemianopia.

https://thejns.org/doi/abs/10.3171/2017.8.JNS171168

KEYWORDS neuromonitoring; visual field; prognostic values; surgical technique

$\mathrm{R}$ ESECTION of intrinsic brain tumors that are close to either the optic nerves, visual tracts, or occipital cortices can cause deterioration of visual function. Typically, these complications occur in procedures for tumors of or around optic nerves ${ }^{4}$ and in temporal lobectomies either for epilepsy surgery ${ }^{1,7,17,18}$ or for temporal neoplasms. ${ }^{2}$ Brain surgeries for parietal and occipital lesions associated with the optic radiations or the visual cortex also carry an intrinsic risk of visual impairment. ${ }^{19}$ Whereas intraoperative motor monitoring has been largely integrated into neurosurgical procedures, ${ }^{9,12,20}$ monitoring of vision has not yet been widely adopted, even though deficits like homonymous hemianopia can significantly impact quality of life and lead, for instance, to a driver's license suspension, reading difficulties, or loss of employment. ${ }^{4}$

The technical feasibility of intraoperative visual evoked potential (VEP) recordings ${ }^{3,16}$ with adequate anesthesia ${ }^{15}$ was previously demonstrated, and flash VEPs have therefore been proposed for visual monitoring during surgical procedures by Kodama and colleagues ${ }^{8}$ and by Sasaki and colleagues..$^{14}$ Indeed, flash VEP monitoring was shown to be predictive of new postoperative visual deficits, and tem-

ABBREVIATIONS AVM = arteriovenous malformation; DNET = dysembryoplastic neuroepithelial tumor; ERG = electroretinogram; LED = light-emitting diode; VEP = visual evoked potential.

SUBMITTED May 12, 2017. ACCEPTED August 28, 2017.

INCLUDE WHEN CITING Published online March 30, 2018; DOI: 10.3171/2017.8.JNS171168. 
porary decreases in VEPs may have contributed to prevent such deficits. In these studies, the alarm criterion was set as a decrease in amplitude of the VEP N2-P2 wave of 50\% or more. With this alarm criterion, new quadrantanopias were not detected. Those studies ${ }^{8,14}$ applied simultaneous electroretinograms (ERGs) and used red light-emitting diodes (LEDs), which stimulate only the cones located in the eye's macula. We hypothesized that the use of white LEDs could activate a larger area of the retina, i.e., all cones and rods, thereby entailing a larger activation of the visual pathways and the occipital cortex. ${ }^{6}$ One potential disadvantage of partial retinal stimulation covering only the macula is that the corresponding coverage of optic fibers along the optic nerve, tract, and radiations, as well as the occipital cortex, could be restricted anatomically, and therefore damage to visual fibers projecting peripheral fields would be underrecognized.

The objective of this study was to assess the performance of white light-induced VEPs in predicting new visual deficits by applying an alarm criterion defined as a reproducible and permanent change of $20 \%$ or more in the amplitude of the VEP N2-P2 wave for stable ERGs.

\section{Methods \\ Patient Population}

This study included 31 patients who underwent craniotomy for the resection of a brain lesion located close to the visual pathways or associated areas. This study was accepted by the Geneva Ethics Committee on Human Research as a prospective study, and it was stopped when VEPs were considered as an effective method for routine intraoperative monitoring. It was conducted according to the ethical guidelines of the Declaration of Helsinki. All patients provided signed consent for their participation.

One patient was excluded from the analyses because of postoperative cerebral ischemia resulting in new hemianopia that occurred after the end of intraoperative VEP monitoring. Another patient was excluded from the analyses for being unable to perform the postoperative visual field assessment. Accordingly, intraoperative monitoring VEPs were analyzed in 29 patients ( 15 female and 14 male patients; median age 46 years; interquartile range 34-61 years; minimum age 17 years, maximum age 77 years). Surgical indications were temporal, temporoinsular, parietal, or parietooccipital lesions (22 patients, $75.9 \%$; Table 1) of diverse origins-gliomas (14 patients), dysembryoplastic neuroepithelial tumors (DNETs; 3 patients), metastases ( 3 patients), or arteriovenous malformations (AVMs; 2 patients) - or frontobasal meningiomas (7 patients, 24.1\%).

\section{Visual Assessment}

The visual field was assessed for all patients preoperatively and at 1 month postoperatively with the Goldmann perimetry test (Octopus; Haag-Streit) by the ophthalmology department of our institution. Visual outcomes were measured as postoperative changes of visual fields and were categorized as "no change," "discrete quadrantanopia" (i.e., new diffuse deterioration $<2 \mathrm{~dB}$ ), "quadrantanopia," "hemianopia," and "blindness."
TABLE 1. Distribution of patients according to location and origin of cerebral lesions

\begin{tabular}{llc}
\hline \multicolumn{1}{c}{ Location of Lesion } & Origin of Lesion & No. of Patients \\
\hline Temporal or temporoinsular & Glioma & 9 \\
& DNET & 3 \\
& AVM & 2 \\
\hline Parietal or parietooccipital & Glioma & 5 \\
& Metastases & 3 \\
\hline Frontobasal & Meningioma & 7 \\
\hline
\end{tabular}

\section{Intraoperative Monitoring of VEPs}

The NimEclipse neuromonitoring system (Medtronic) was used to elicit flash VEPs. This system provides goggles equipped with white LEDs. The goggles, which were placed over the patients' eyes, were carefully protected from scialytic operating room lamps and from the microscope light with a film of aluminum. A soft tissue was used to separate the aluminum and the patient's skin (Fig. 1).

The amplitude of the flash VEP wave was monitored with the peak-to-peak amplitude of N2-P2 waves..$^{13}$ Occipital subcutaneous corkscrew electrodes were placed to record the VEPs (DME1001; Medtronic Xomed, Inc.). The localizations were $\mathrm{Oz}, \mathrm{O} 1, \mathrm{O} 2$ with preferred reference at the vertex, and $\mathrm{Cz}$, with the ground electrode made of linked A1 and A2 (international 10/20 EEG system). All wires were carefully twisted together to improve signal-to-noise ratios of recordings. Signals were averaged over typically 50 sweeps for every VEP (bandpass filtering 2-400 Hz, length $200 \mathrm{msec}$ ). Occipital subdural strip electrodes were used in cases of occipital brain lesions (MS04N-IP10X-0JF; Ad-Tech). The duration of the stimulation was $8 \mathrm{msec}$ of white light, as recommended for ERGs ${ }^{11}$ with a luminescence of 500-10,000 Lx, at a rate of $0.9 \mathrm{~Hz}$. The amplitude of ERGs was monitored with peak-to-peak amplitude. ${ }^{11}$ Left and right eyes were excited sequentially, and VEPs were performed for one eye at a time, alternating periods of VEP recordings for the other

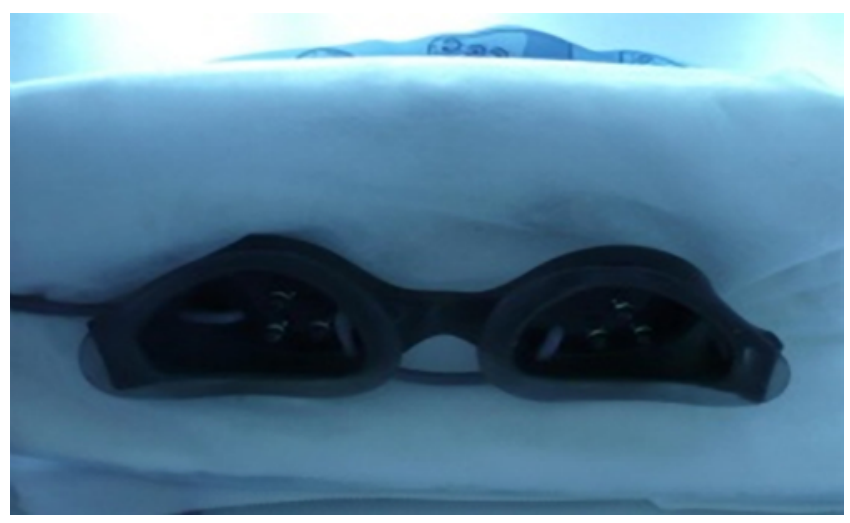

FIG. 1. Goggles equipped with white LEDs (NimEclipse; Medtronic) were placed over the patient's eyes and protected from the lights of the operating room by using aluminum film. A soft tissue is used to separate the aluminum from the patient's skin. 
eye. According to the quality of VEPs, in particular with regard to the protection from the light of the operating room obtained for every eye, a single eye was in practice preferentially measured.

The ERGs were recorded with a pair of subdermal electrodes placed laterally to every patient's eye (DSN2282; Medtronic Xomed, Inc.). Both wires were carefully twisted together to improve signal-to-noise ratios. Changes in ERGs could either occur with dark or light adaptation ${ }^{11}$ or be due to displacements of goggles or of their aluminum protection. A new VEP baseline was obtained at the beginning of the lesion resection after the microscope was brought into place.

\section{Alarm Criteria}

The VEP baselines were updated with ERG changes, as can be expected from changes in rod and cone excitation with repetitions of white flash VEPs. ${ }^{11}$ The alarm was defined as a reproducible $20 \%$ decrease or more in the peakto-peak amplitude of N2-P2 waves, with concomitant stable ERGs. In such case, the alarm was communicated to the surgeon.

The evaluation of proportions (sensitivity and specificity) and performances (positive and negative predictive values) was defined as true negative (no permanent decrease in VEP amplitude and no new postoperative visual deficit); true positive (permanent decrease in VEP and new postoperative visual deficit); false negative (no permanent decrease in VEP, but new postoperative visual deficit); and false positive (permanent decrease in VEP, but no new postoperative visual deficit).

\section{Anesthesia Protocol}

Target-controlled infusion of propofol or sufentanil was used to induce anesthesia (maintenance concentration according to the patient's needs, with propofol $3.0[4.5 \mu \mathrm{g} /$ $\mathrm{ml}]$ or sufentanil $0.15[0.25 \mathrm{ng} / \mathrm{ml}])$, following recommendations from Wiedemayer and colleagues. ${ }^{15}$

\section{Results}

Flash VEPs were measured for 51 eyes in 29 patients. Stable and reliable VEPs with ERGs were obtained in $80.4 \%$ (41/51) of eyes, for $89.7 \%$ (26/29) of patients. Noncontributive VEPs were due to either unstable VEPs or unstable ERGs, generally attributed to displacements of the goggles or to the aluminum film protection. These noncontributive VEPs were encountered for both eyes in 3 patients, who were excluded from the analyses.

An example of VEP recordings is provided in Fig. 2. The VEP recordings from the left occipital electrodes are illustrated in the first column, and from the right occipital electrodes in the second column (here for the excitation of the right eye). The simultaneous ERGs are found in the third column. The amplitudes of the N2-P2 waves of VEP for both hemispheres were monitored, as were the amplitudes of the $a-b$ waves of the ERG (the alarm was only triggered when VEP amplitude decreased while ERGs were stable).

In $65.4 \%(17 / 26)$ of patients no new postoperative visual field deficits were observed, and in $73.2 \%$ (30/41) of eyes no reproducible decreases of more than $20 \%$ in VEP amplitudes were recorded (true negative; Table 2).

In 1 case (i.e., 3.8\% [1/26]) of patients and 4.9\% (2/41) of eyes, no new postoperative visual deficit was observed, but a reproducible VEP decrease of more than $20 \%$ was measured (false positive). In this particular case the patient already had a temporal heteronymous hemianopia prior to surgery due to chiasm compression from a suprasellar meningioma. The decrease in VEP was attributed to additional manipulation of the optic chiasma during surgery without clinical increase of visual field deficits. Hence, the absence of an alarm was associated with the absence of postoperative visual changes in $94.4 \%$ (17/18) of patients and in $93.8 \%$ (30/32) of all eyes (specificity; Table 2).

In $19.2 \%(5 / 26)$ of patients, new postoperative deficits were observed, and a permanent decrease of more than $20 \%$ in VEP amplitudes was recorded in $14.6 \%$ (6/41) eyes (true positive). A new quadrantanopia occurred in 1 patient with temporal lobe DNET. In this case the VEP deterioration was not considered critical enough to decide to modify this surgery performed to cure pharmacoresistant epilepsy, which necessitates extended resection. A deterioration of a preexistent quadrantanopia was observed in another patient during temporoinsular resection. In that case, VEP was not performed frequently enough because of simultaneous motor neuromonitoring, and the VEP alarm was provided with some delay. Finally, deterioration of a preexistent hemianopia occurred in 3 cases: 1 case with temporoinsular resection and late VEP alarm, and 2 meningioma cases; VEPs deteriorated abruptly in one of each (temporoinsular lesion and meningioma) at the time of hemostasis, and at the time of optic tract manipulation for the other (meningioma). Hence, the alarms predicted postoperative visual impairments in $83.3 \%(5 / 6)$ of patients (i.e., 75\% [6/8] of eyes-positive predictive value; Table 2).

In $11.5 \%$ (3/26) of patients and 7.3\% (3/41) of eyes, new postoperative deficits were observed, but reproducible decreases in VEP amplitude remained below 20\% (false negative). Hence, the alarms correctly identified $62.5 \%$ (5/8) of patients (i.e., 66.7\% [6/9] of eyes) with postoperative visual changes (sensitivity). The absence of an alarm predicted an absence of postoperative visual impairment in $85.0 \%(17 / 20)$ of patients and in $90.9 \%$ (30/33) of eyes (negative predictive value; Table 2).

The relatively low sensitivity was due to 3 cases with either new discrete homonymous quadrantanopia or deterioration of an existing quadrantanopia (Fig. 3). Indeed, taking this into account, the alarm threshold-defined as a reproducible 20\% decrease in VEP amplitude-identified $100 \%$ of all eyes with postoperative visual changes more severe than just a discrete quadrantanopia.

Temporary decreases in VEP amplitude of $20 \%$ or more that fully recovered during surgery were encountered in $11.5 \%$ of patients (3/26) and in 9.8\% (4/41) of eyes. None of these patients presented with new visual deficits. One episode of temporary VEP loss was observed during amygdalohippocampectomy in a DNET case, triggering the temporary interruption of surgery and a repeated check of the anatomical landmarks before continuing with the hippocampectomy. In this case, the preoperative ophthalmological examination showed superior homonymous 

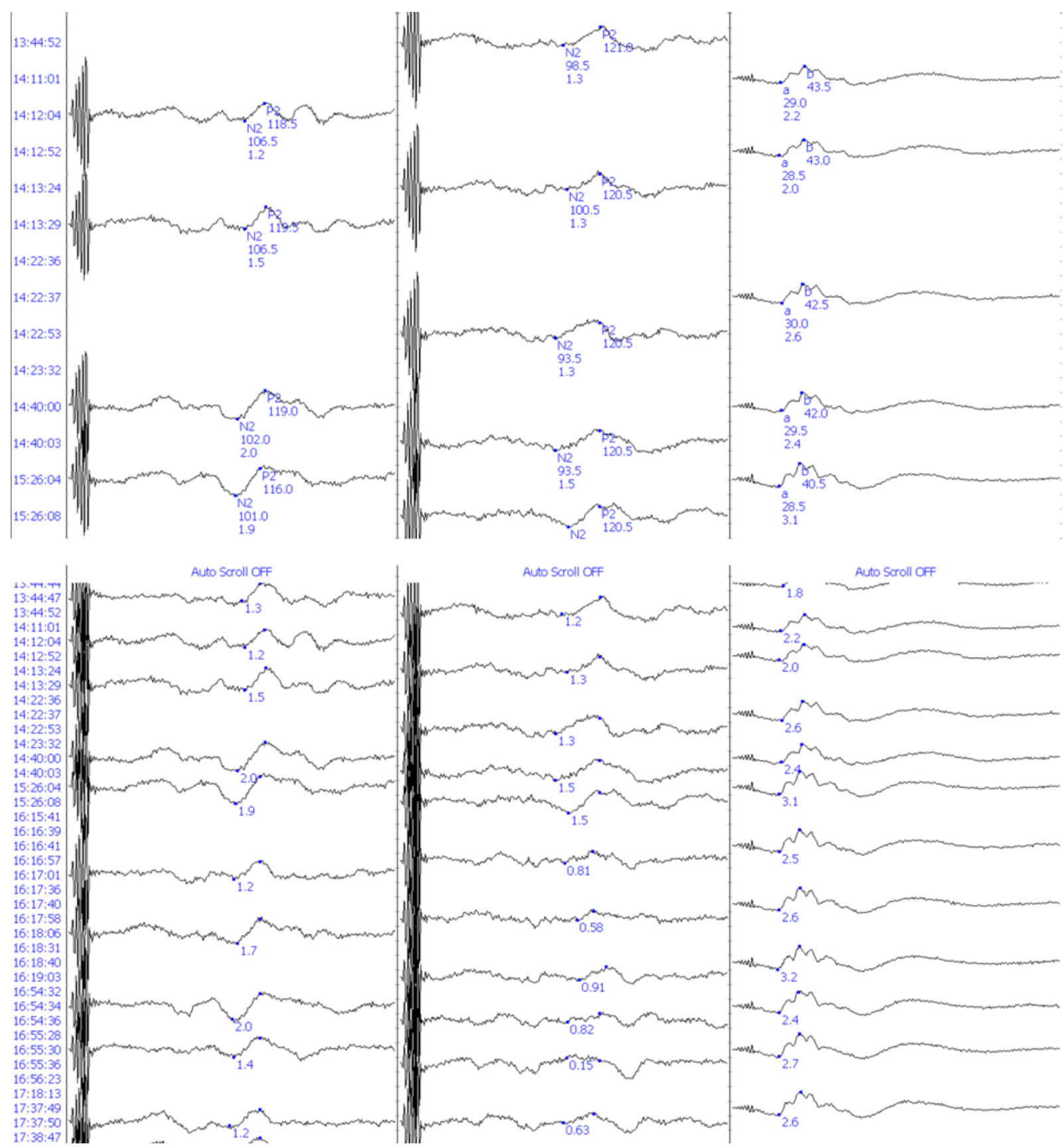

FIG. 2. Upper: Detailed VEP measurements, with latencies and peak-to-peak amplitudes of the N2-P2 waves, in the first column for left occipital recordings, and in the second column for right occipital recordings. The last column shows ERGs with a-b waves, completed by their latencies and peak-to-peak amplitude. Lower: Example of VEP monitoring: the alarm was triggered only when the N2-P2 amplitude of the VEPs decreased (right occipital recordings, second column), whereas the ERGs were stable (last column).

quadrantanopia, and postoperative ophthalmological assessment at 1 month showed no new visual field deficit. In another patient with suprasellar meningioma (Simpson grade II), a temporary VEP loss was observed at the time of manipulation of the optic chiasma, but no visual change was observed postoperatively. In the remaining patient, the temporary decrease was seen during glioma resection in the parietal lobe. Again, subsequent to the VEP alarm, anatomical landmarks were rechecked before continuing with the resection, which did not result in new postoperative visual field deficit.

\section{Discussion}

This is the first study to assess the performance of intraoperative VEPs performed with white LEDs with simultaneous recordings of ERGs. Here the alarm criterion was a VEP amplitude decrease of $20 \%$ or more. This 
TABLE 2. Distribution of cases obtained with an alarm criterion determined as a reproducible VEP amplitude decrease of $20 \%$ or more

\begin{tabular}{|c|c|c|c|c|c|}
\hline Finding & No. of Patients & No. of Eyes & $\begin{array}{c}\text { Distribution of VEP } \\
\text { Decreases (no. of eyes) }\end{array}$ & $\begin{array}{l}\text { Postop Changes in } \\
\text { Visual Fields }\end{array}$ & Site of Surgery \\
\hline True negative & 17 & 30 & $\leq 20 \%(29)$ & None & \\
\hline False positive & 1 & 2 & $100 \%(2)$ & None, chiasma manipulation & Frontobasal \\
\hline \multirow[t]{2}{*}{ False negative } & \multirow[t]{2}{*}{3} & \multirow[t]{2}{*}{3} & $0 \%(2)$ & \multirow[t]{2}{*}{ Discrete quadrantanopia } & Temporoinsular, temporomesial \\
\hline & & & $17 \%(1)$ & & Temporoinsular \\
\hline \multirow[t]{4}{*}{ True positive } & \multirow[t]{4}{*}{5} & \multirow[t]{4}{*}{6} & $25 \%(1)$ & Quadrantanopia & Frontobasal \\
\hline & & & $80 \%(1)$ & \multirow[t]{2}{*}{ Hemianopia } & \multirow[t]{2}{*}{ Temporoinsular } \\
\hline & & & $90 \%(1)$ & & \\
\hline & & & $100 \%(3)$ & Blindness or hemianopia & Frontobasal, frontobasal, temporal \\
\hline Total & 26 & 41 & & & \\
\hline
\end{tabular}

Discrete quadrantanopia was defined as new diffuse deterioration $<2 \mathrm{~dB}$.

alarm criterion allowed us to detect visual field changes larger than a discrete quadrantanopia, or a deterioration of an existing quadrantanopia (Fig. 3). In previous studies the alarm criterion was determined as a VEP amplitude decrease of $50 \%$ using red LEDs. ${ }^{8,14}$ In a series of 53 consecutive patients, Kodama and colleagues ${ }^{8}$ did not detect new quadrantanopias. This could be explained by an alarm criterion of 50\%, which is higher than the one used in the present study (i.e., 20\%). Moreover, Kodama and colleagues encountered severe new visual field deficits in $7.8 \%$ of eyes, which is lower than those encountered in the present cohort $(12.2 \%, 5 / 41$ eyes, for hemianopia and blindness; Table 1). However, these percentages cannot be properly compared, because the detailed ratio of different surgical indications was not described in the study of Kodama and colleagues, and because patients undergoing operations for aneurysms were not included in our present study. In another study, Sasaki and colleagues ${ }^{14}$ reported new blindness, hemianopias, or quadrantanopias in $12.1 \%$ (i.e., $7 / 58$ ) of patients who underwent operations for tumors or AVMs, which is similar to our results. Note that because patients who were surgically treated for aneurysms were not included in our study, the global sensitivity and specificity of VEP alarms cannot be compared with those reported by Sasaki and colleagues. In a third study, Luo and colleagues ${ }^{10}$ reported low sensitivity and specificity of red-light VEPs, which may be explained by the lack of ERG monitoring in their study.

Finally, both Kodama and colleagues ${ }^{8}$ and Sasaki and colleagues ${ }^{14}$ have also described episodes of transient decrease in VEPs without associated postoperative visual field deficits. In our series, similar cases were rarely encountered (3 cases). To detect very early changes in VEP amplitudes and hence to provide an alarm early enough, improved techniques are required to decrease the time necessary to get each averaged VEP.

Kodama and colleagues ${ }^{8}$ and Sasaki and colleagues ${ }^{14}$ reported a success rate for VEP monitoring of $97 \%$ (103/106) and 93.5\% (187/200) of eyes, respectively. Ours is lower, with a ratio of $80.4 \%$ (41/51) of eyes. In this study,
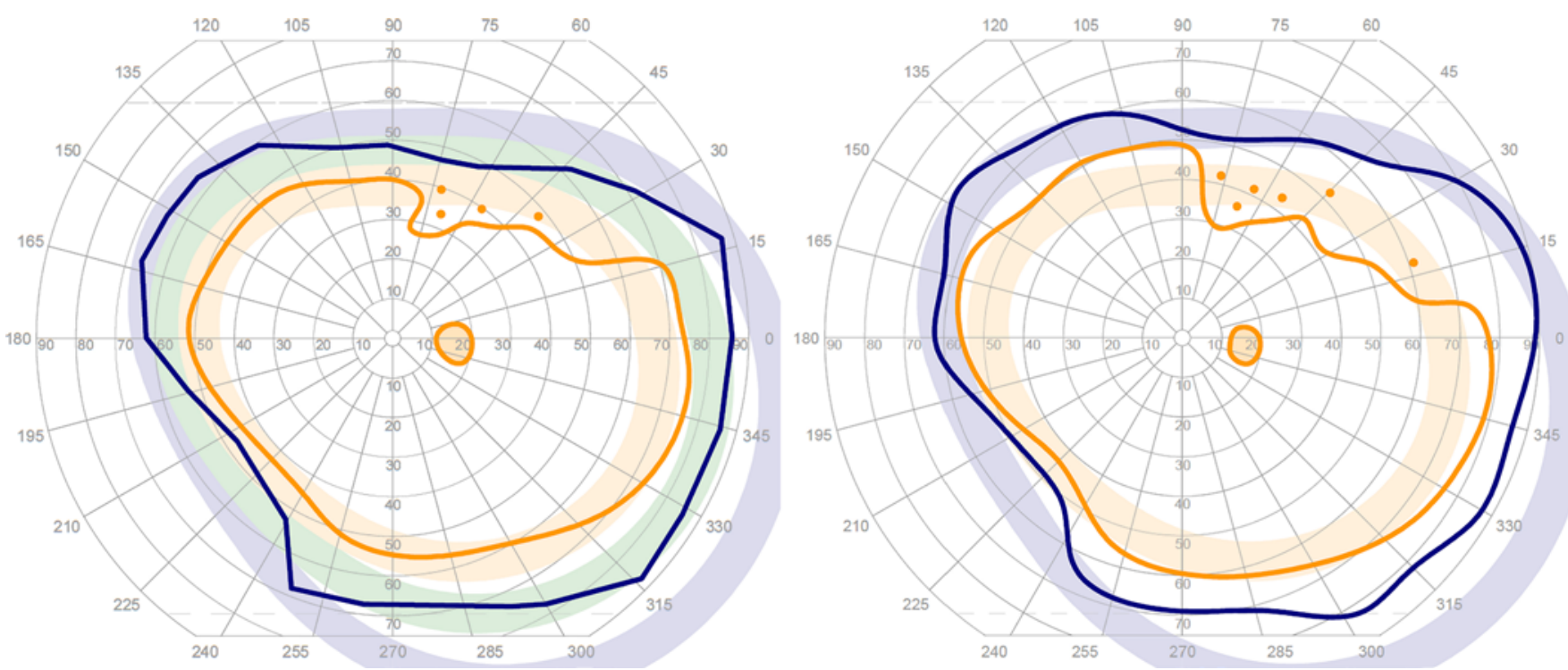

FIG. 3. Preoperative (left) and postoperative (right) Goldmann perimetry in 1 patient who underwent insular glioma resection. The quadrantanopia deterioration of the right eye was not detected by VEP amplitude decreases of more than $20 \%$. 
a film of aluminum, separated from the patient's skin with tissue, was applied to protect the goggles against the light of the surgery room, i.e., from the scialytic operating room lamps and the microsurgical microscope, which can prevent possible VEP deterioration if interfering with the LEDs. ${ }^{5}$ Getting stable light exposure with stable LEDs, and with stable protection from the lights of the operative field per se, is certainly an important point in acquiring stable VEPs. Implementation of VEPs would be improved again with advanced LEDs, protecting the eyes from light exposure changes, due to either the light of the operating room or goggle displacements.

To improve VEP performance, we hypothesized that the white flash light would stimulate all cones in the macula, because white light contains all colors of the light spectrum, therefore potentially increasing the spatial resolution of macular coverage. In addition, because white flashes also excite rods, we speculated that rod stimulation would increase the spatial coverage of visual field pathways and occipital cortex by stimulating the extramacular peripheral retina, which is rich in rods and poor in cones. Excitation of rods or cones can actually be determined from the waveform of the ERG. ${ }^{11}$ Although red and white LEDs were not compared head-to-head, it is nevertheless plausible that white LEDs could provide better monitoring of visual fields, possibly facilitating the detection of quadrantanopia.

\section{Limitations of the Study}

The patients' visual acuity has not been included in this study. With $0.9-\mathrm{Hz}$ white flash stimuli, repeated all throughout the surgeries, not only cone or rod excitation can be expected. ${ }^{11}$ The possible changes in ERG amplitudes and waveforms due to possible retinal excitation changes were carefully measured to be integrated into the analysis of the changes in the VEP amplitudes. The placement per se of the goggles with their aluminum protection film did not allow VEP monitoring for anterochiasmal procedures.

\section{Conclusions}

This study demonstrates the effectiveness and reliability of intraoperative monitoring with VEPs performed using white LEDs during brain lesion resection near the visual tracts or areas. With an alarm criterion defined as a reproducible decrease in VEP amplitude of $20 \%$ or more, intraoperative monitoring of VEPs can predict new postoperative visual deficits that are more severe than just a discrete quadrantanopia or deterioration of an existing quadrantanopia. In addition, full intraoperative recovery of VEPs after an initial decrease, and subsequent corrective surgical action in a few cases, may suggest that VEP monitoring can contribute to protect visual function. More experience in this technique is needed to adapt surgical strategy for the preservation of vision.

Finally, technical improvements, such as optimized LED goggle design and robust protection from surrounding operating room lights, would certainly facilitate the routine application of VEPs in a daily intraoperative setting. This could be done on par with motor and sensory evoked potentials, offering a useful additional tool to the surgeon and a benefit to patients.

\section{Acknowledgments}

We thank D. Conan and C. Bech, nurse and technician, respectively, dedicated to intraoperative neuromonitoring; and C. Sauvadet and P. Sanchette, both ophthalmological technicians, who performed all perimetry examinations.

\section{References}

1. Chen X, Weigel D, Ganslandt O, Buchfelder M, Nimsky C: Prediction of visual field deficits by diffusion tensor imaging in temporal lobe epilepsy surgery. Neuroimage 45:286-297, 2009

2. Faust K, Vajkoczy P: Distinct displacements of the optic radiation based on tumor location revealed using preoperative diffusion tensor imaging. J Neurosurg 124:1343-1352, 2016

3. Feinsod M, Selhorst JB, Hoyt WF, Wilson CB: Monitoring optic nerve function during craniotomy. J Neurosurg 44:29_ 31, 1976

4. Goodwin D: Homonymous hemianopia: challenges and solutions. Clin Ophthalmol 8:1919-1927, 2014

5. Huang S, Hokenson K, Bandyopadhyay S, Russek SJ, Kirkwood A: Brief dark exposure reduces tonic inhibition in visual cortex. J Neurosci 35:15916-15920, 2015

6. Hubel DH, Wiesel TN: Brain mechanisms of vision. Sci Am 241:150-162, 1979

7. Jeelani NU, Jindahra P, Tamber MS, Poon TL, Kabasele P, James-Galton M, et al: 'Hemispherical asymmetry in the Meyer's Loop': a prospective study of visual-field deficits in 105 cases undergoing anterior temporal lobe resection for epilepsy. J Neurol Neurosurg Psychiatry 81:985-991, 2010

8. Kodama K, Goto T, Sato A, Sakai K, Tanaka Y, Hongo K: Standard and limitation of intraoperative monitoring of the visual evoked potential. Acta Neurochir (Wien) 152:643648,2010

9. Kombos T, Suess O, Ciklatekerlio O, Brock M: Monitoring of intraoperative motor evoked potentials to increase the safety of surgery in and around the motor cortex. J Neurosurg 95:608-614, 2001

10. Luo Y, Regli L, Bozinov O, Sarnthein J: Clinical utility and limitations of intraoperative monitoring of visual evoked potentials. PLoS One 10:e0120525, 2015 (Erratum in PLoS One 10:e 0133819, 2015)

11. McCulloch DL, Marmor MF, Brigell MG, Hamilton R, Holder GE, Tzekov R, et al: ISCEV Standard for full-field clinical electroretinography (2015 update). Doc Ophthalmol 130:1-12, 2015

12. Neuloh G, Pechstein U, Schramm J: Motor tract monitoring during insular glioma surgery. J Neurosurg 106:582-592, 2007

13. Odom JV, Bach M, Brigell M, Holder GE, McCulloch DL, Mizota A, et al: ISCEV standard for clinical visual evoked potentials: (2016 update). Doc Ophthalmol 133:1-9, 2016

14. Sasaki T, Itakura T, Suzuki K, Kasuya H, Munakata R, Muramatsu $\mathrm{H}$, et al: Intraoperative monitoring of visual evoked potential: introduction of a clinically useful method. J Neurosurg 112:273-284, 2010

15. Wiedemayer H, Fauser B, Armbruster W, Gasser T, Stolke D: Visual evoked potentials for intraoperative neurophysiologic monitoring using total intravenous anesthesia. J Neurosurg Anesthesiol 15:19-24, 2003

16. Wilson WB, Kirsch WM, Neville H, Stears J, Feinsod M, Lehman RA: Monitoring of visual function during parasellar surgery. Surg Neurol 5:323-329, 1976

17. Yeni SN, Tanriover N, Uyanik O, Ulu MO, Ozkara C, Karaağaç N, et al: Visual field defects in selective amygdalohippocampectomy for hippocampal sclerosis: the fate of Meyer's loop during the transsylvian approach to the temporal horn. Neurosurgery 63:507-515, 2008 
18. Yogarajah M, Focke NK, Bonelli S, Cercignani M, Acheson J, Parker GJ, et al: Defining Meyer's loop-temporal lobe resections, visual field deficits and diffusion tensor tractography. Brain 132:1656-1668, 2009

19. Zhang X, Kedar S, Lynn MJ, Newman NJ, Biousse V: Homonymous hemianopia in stroke. J Neuroophthalmol 26:180183,2006

20. Zhou HH, Kelly PJ: Transcranial electrical motor evoked potential monitoring for brain tumor resection. Neurosurgery 48:1075-1081, 2001

\section{Disclosures}

Mrs. Boëx has taught at workshops organized by Medtronic.

\section{Author Contributions}

Conception and design: Boëx, Gutzwiller, Cabrilo, Radovanovic.
Acquisition of data: Boëx, Gutzwiller, Schaller. Analysis and interpretation of data: Boëx, Gutzwiller. Drafting the article: Boëx, Gutzwiller. Critically revising the article: Boëx, Cabrilo, Radovanovic, Schaller. Reviewed submitted version of manuscript: all authors. Approved the final version of the manuscript on behalf of all authors: Boëx. Administrative/technical/material support: Gutzwiller. Support of the study: Schaller.

\section{Supplemental Information}

Previous Presentations

Portions of this work were presented in poster forms at proceedings of the 15th European Congress of Neurosurgery, Prague, Czech Republic, held in October 2014.

\section{Correspondence}

Colette Boëx: University Hospital of Geneva, Switzerland. colette. boex@hcuge.ch. 